\title{
Posterior ocular abnormalities after irradiation for retinoblastoma: a histopathological study
}

\author{
PETER R. EGBERT, ${ }^{1}$ LUIS F. FAJARDO,${ }^{2}$ SARAH S. DONALDSON,${ }^{3}$ \\ AND KAMBIZ MOAZED ${ }^{1}$ \\ From the ${ }^{1}$ Department of Surgery, Division of Ophthalmology, Stanford University Medical Center, \\ Stanford, CA; the ${ }^{2}$ Department of Pathology, Stanford University School of Medicine and \\ Veterans Administration Medical Center, Palo Alto, CA; and the ${ }^{3}$ Department of Radiology, \\ Division of Radiation Therapy, Stanford University Medical School, Stanford, CA, USA
}

SUMMARY Radiation-induced ocular lesions in the posterior eye and orbit ware investigated in 33 surgical specimens of patients with retinoblastoma. The eyes were obtained from children 7 months to 6 years of age. Seventeen eyes were irradiated; 16 eyes had not received irradiation and served as controls. The majority of the irradiated eyes were treated with 6000 rads of external beam radiation. They were removed at a mean of 23 months after radiotherapy. All specimens were examined simultaneously by 2 observers without knowledge of treatment and analysed for the presence or absence of 15 lesions. The most consistent lesions in the irradiated eyes were abnormalities of the retinal vessels ( 11 of 17 eyes) and striking changes in the ciliary arteries (13 of 17 eyes). The retinal vessels showed thickening of the wall, often caused by deposition of fibrillary material, sometimes with fibrin deposits. The most consistent lesion was myointimal proliferation with narrowing of the ciliary arteries. Lesions of the central retinal artery were less common but occurred only in irradiated patients.

Radiotherapy is effective and valuable for diseases in or near to the eye; however, its usefulness is limited by the possibility of ocular radiation injury. Damage to the anterior structures of the eye, especially the lens and cornea, by low doses of radiation (in the range of 1000 rads) has been well documented, ${ }^{1}$ whereas it has been considered that the posterior parts of the eye tolerate higher doses. ${ }^{2}$ Damage to the retina and optic nerve can occur with fractionated radiation above 6800 rads. ${ }^{3}$ Changes in the ciliary vessels have not, to our knowledge, been described. We have examined the histological changes in a series of eyes that were removed after external radiotherapy for retinoblastoma and have found damage in the posterior ciliary vessels, optic nerve, and retinal vessels.

\section{Materials and methods}

Thirty-three eyes from patients with retinoblastoma were examined; 17 eyes had been irradiated and subsequently enucleated, and 16 eyes had not been

Correspondence to Peter R. Egbert, MD, Stanford University Medical Center, Boswell Building, Room A-227, Stanford, California 94305 , USA. irradiated and served as controls. All specimens were examined simultaneously by 2 observers (PRE, LFF) in a doutle-head microscope, without knowledge of treatment, and analysed for the presence or absence of 15 morphological abnormalities. The severity of myointimal proliferation of the posterior ciliary arteries was graded in a scale of 0 (normal) to 4 (complete luminal obstruction). Five normal eyes from necropsies of children were also examined for the same lesions.

Standard axial sections of the entire eyeball emtedded in paraffin as well as transverse sections of the optic nerve were used. In addition to haematoxylin and eosin the stains used included periodic acid Schiff (PAS), Verhoeff's elastic (EVG), FraserLendrum method for fibrin, Alcian blue method for acid mucopolysaccharides, Luxol-fast blue for myelin, and the Bielschowsky method for nerve fibres.

The irradiated eyes had been enucleated because of uncontrolled tumour growth, pain from neovascular glaucoma, or inability to examine the eye because of vitreous haemorrhage. Most of the eyes came from patients who were part of a previous study of visual function following radiotherapy. ${ }^{4}$ 
The irradiated eyes had been treated by megavoltage irradiation generated from a $6 \mathrm{MeV}$ medical linear accelerator with doses of 5200 to 6000 rads given in 200-250 rad fractions over approximately 6 weeks. The one exception to this dose occurred in an eye enucleated 2 weeks after initiation of treatment, having received only 2250 rads. Five eyes of 5 patients had more than 1 course of radiation, which significantly increased the total dose to as much as 13000 rads; 4 of these patients were also given intra-arterial triethylenemelamine and 1 eye had a ${ }^{125}$ I implant. Several eyes were treated by photocoagulation or cryotherapy.

With the exception of one 6-year-old child, the patients' ages at the beginning of radiotherapy ranged from 7 months to 2 years. The age range of the nonirradiated controls was comparable. The interval from the first day of radiotherapy to enucleation was 2 weeks to 34 months (mean 23 months).

\section{Results}

There were clear differences between the irradiated and nonirradiated eyes (Table 1). The most consistent changes were in retinal vessels and extraocular posterior ciliary arteries. Cataract and neovascularisation of the iris were also seen in irradiated eyes. However, the comments that follow refer only to the important lesions in the posterior part of the eye.

Retinal vessel changes were seen in 11 of 17 irradiated eyes. Marked thickening of the arteriolar and capillary walls occurred, both in residual tumour and in areas away from the tumour. Most often the thickening was caused by the accumulation in the entire wall of a fine fibrillary material

Table 1 Posterior ocular histological changes associated with radiotherapy

\begin{tabular}{|c|c|c|c|}
\hline & \multicolumn{2}{|c|}{ Retinoblastoma } & \multirow{2}{*}{ Normal } \\
\hline & Radiation & No radiation & \\
\hline Number of eyes & 17 & 16 & 5 \\
\hline $\begin{array}{l}\text { Lesions of retinal vessels } \\
\text { (away from tumour) }\end{array}$ & 11 & 0 & 0 \\
\hline $\begin{array}{l}\text { Myointimal proliferation } \\
\text { in central retinal artery }\end{array}$ & 4 & 0 & 0 \\
\hline $\begin{array}{l}\text { Myointimal proliferation } \\
\text { in posterior ciliary vessels }\end{array}$ & 13 & 0 & 0 \\
\hline $\begin{array}{l}\text { Optic nerve axonal and } \\
\text { myelin loss }\end{array}$ & 13 & 11 & 0 \\
\hline Diffuse & 6 & 1 & \\
\hline Segmental & 1 & 6 & \\
\hline Diffuse and segmental & 6 & 4 & \\
\hline
\end{tabular}
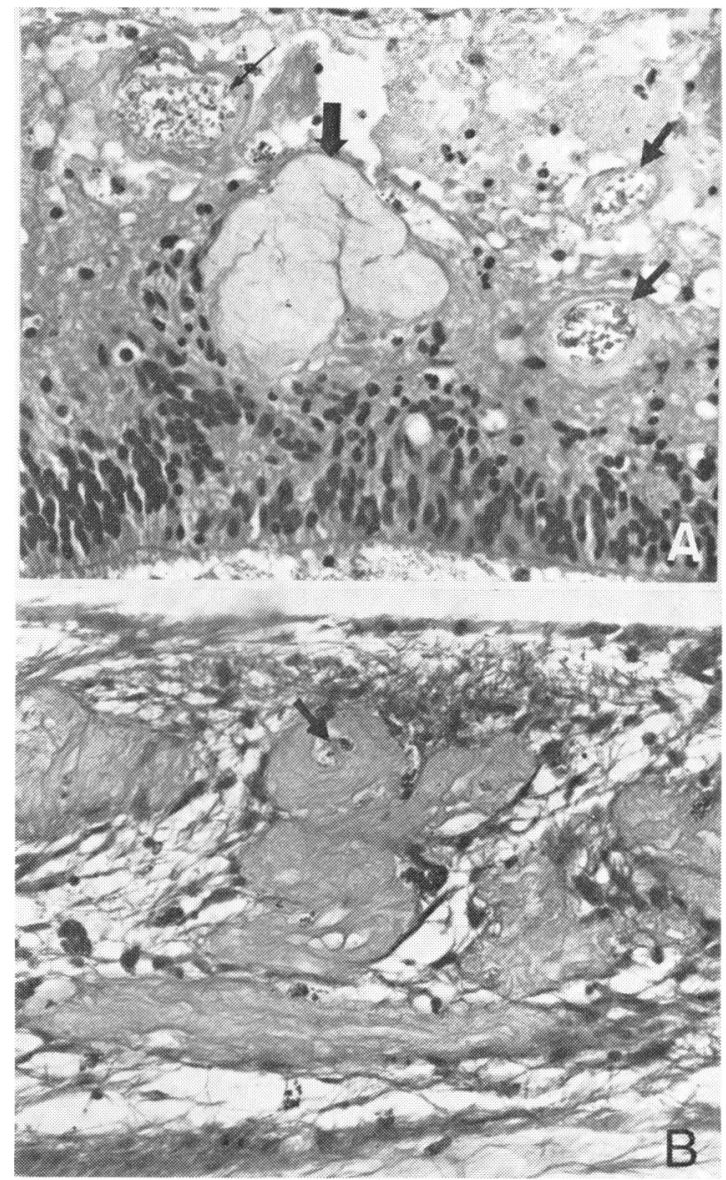

Fig. 1 Perpendicular sections of retina. A: Eye resected 6 months c fter 6000 rads/25 fractions/44 days. Notice marked atrophy of the outermost layers and abnormalities of vessels. There is irregular wall thickening and dilatation of capillaries (small arrows). In the centre (large arrow) is an arteriole totally replaced by convoluted, fibrillary material. Its lumen is no longer recognisable. B: Specimen obtained 7 months after 5950 rads/29 fractions/43 days. There is severe atrophy of all retinal layers. All vessels in this field are markedly thickened by fine fibrillary material which is in or outside the vessel walls. The remaining lumen can be recognised in some (arrow).

(Haematoxylin and eosin, $\times 200$ ).

(PAS-negative) which narrowed the lumen and appeared to progress to occlusion (Figs. 1,2). This material replaces the wall and may be partly extravascular. Fibrin was demonstrated in the wall of a number of vessels by the Fraser-Lendrum or the phosphotungstic acid-haematoxylin (PTAH) stains (Fig. 2). Fibrin was also found outside of the vessels in the adjacent retinal stroma. Extensive vascular 


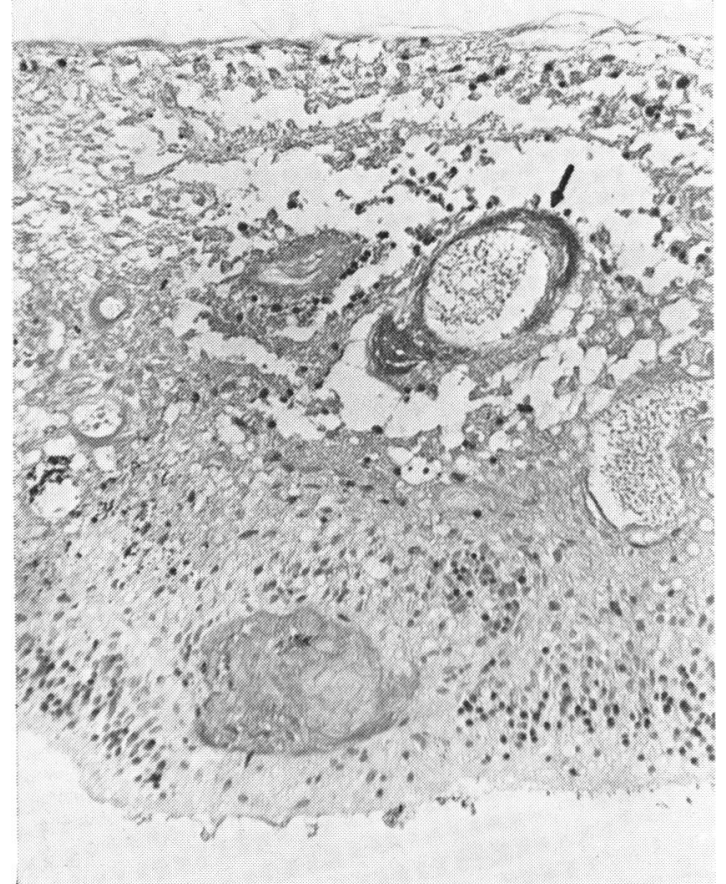

Fig. 2 Section of retina from the same eye described in Fig. 1A. Notice totally obliterated vessel in the outer retina. One of the vessels in the inner layers (arrow) shows not only dilatation but also severe fibrinous exudate in the wall, which appears black (red-purple in the histological preparation).

(Fraser-Lendrum stain, $\times 135$ ).

proliferation into the vitreous was present in 2 eyes. There were no retinal vessel changes in the control nonirradiated eyes.

The posterior ciliary arteries in the region of the anterior optic nerve and posterior sclera showed abnormalities in 13 of the 17 irradiated eyes and in none of the unirradiated eyes. These changes were present as early as 5 months after initiation of irradiation. Myointimal proliferation, either concentric or eccentric, encroached on the lumen of arteries of $100 \mu \mathrm{m}$ to $200 \mu \mathrm{m}$ in diameter and at times obliterated the lumen completely (Figs. 3, 4). Enlarged endothelial cells and thick elastic lamina were also seen (Fig. 4). Although these changes were obvious in the larger ciliary arteries, adjacent veins and venules, as well as smaller arterioles and capillaries, were usually normal. Occasionally similar abnormalities were present in arterioles in the optic nerve sheath or in the optic nerve itself; however, capillaries in the optic nerve usually appeared unremarkable. Myointimal proliferation of the central retinal artery, without occlusion, was found in 4 eyes enucleated $5,12,17$, and 23 months after radiation (Fig. 5).

Optic nerve atrophy was observed in nonirradiated as well as in irradiated eyes. It was characterised by a decrease in the diameter of the nerve and loss of axons and myelin. The distribution of this lesion, however, was not the same in both groups. Diffuse atrophy (Fig. 6) was seen preferentially in the irradiated eyes; a segmental atrophy (Figs. 7,8 ) was seen preferentially in nonirradiated eyes (Table 1).

The choroid was difficult to evaluate because of the large number of collapsed vessels, adjacent to each other; we did not identify obvious choroidal radiation-induced changes. The retinal pigment epithelium showed areas of atrophy and areas of hyperplasia in both irradiated and nonirradiated eyes. The sclera also had no radiation-related abnormalities.

There was no consistent relationship between the severity of myointimal proliferation in the ciliary arteries and the age of the patient, the radiation dose, or the length of the interval from the first day of radiation to enucleation. The 5 eyes which had no changes in the posterior ciliary arteries were enucleated one-half, 5, 7, 7, and 13 months after initiation of radiotherapy. All eyes removed later had changes; however, damage to the posterior ciliary arteries was also seen in 3 eyes removed 12 months or less after radiotherapy.

Vascular injury was seen in eyes at the low as well as the high end of the dose range. One eye showed

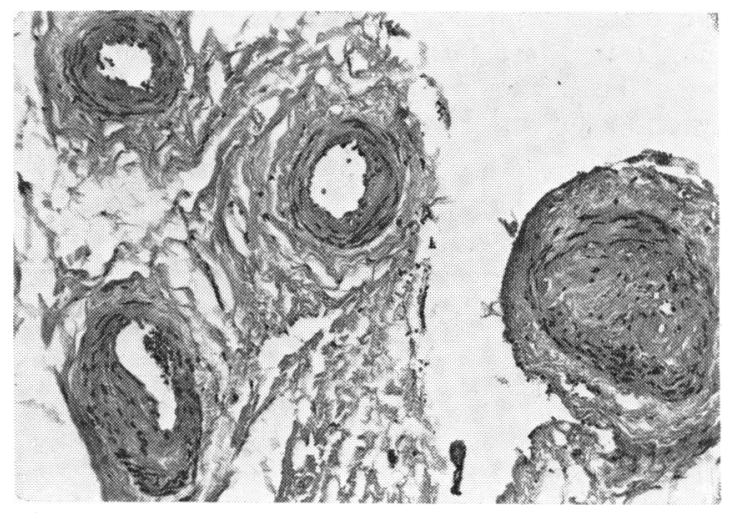

Fig. 3 Transverse sections of posterior ciliary arteries from eye resected 2 years after 6000 rads/25 fractions $/ 44$ days. The arteries in centre and left upper corner are essentially normal. There is eccentric myointimal proliferation with slight narrowing of vessel in left lower corner. The artery on the right is totally occluded by myointimal proliferation. This case illustrates the random distribution of radiation-induced lesions. (Haematoxylin and eosin, $\times 90$ ). 
damage after 5200 rads and several after 6000 rads. All eyes that had multiple courses of radiation had vascular damage, usually severe. The eye enucleated after only 2250 rads ( 2 weeks after the initial dose) showed no vascular damage.

\section{Discussion}

The most interesting findings in this study were the abnormalities in the posterior ciliary arteries. We

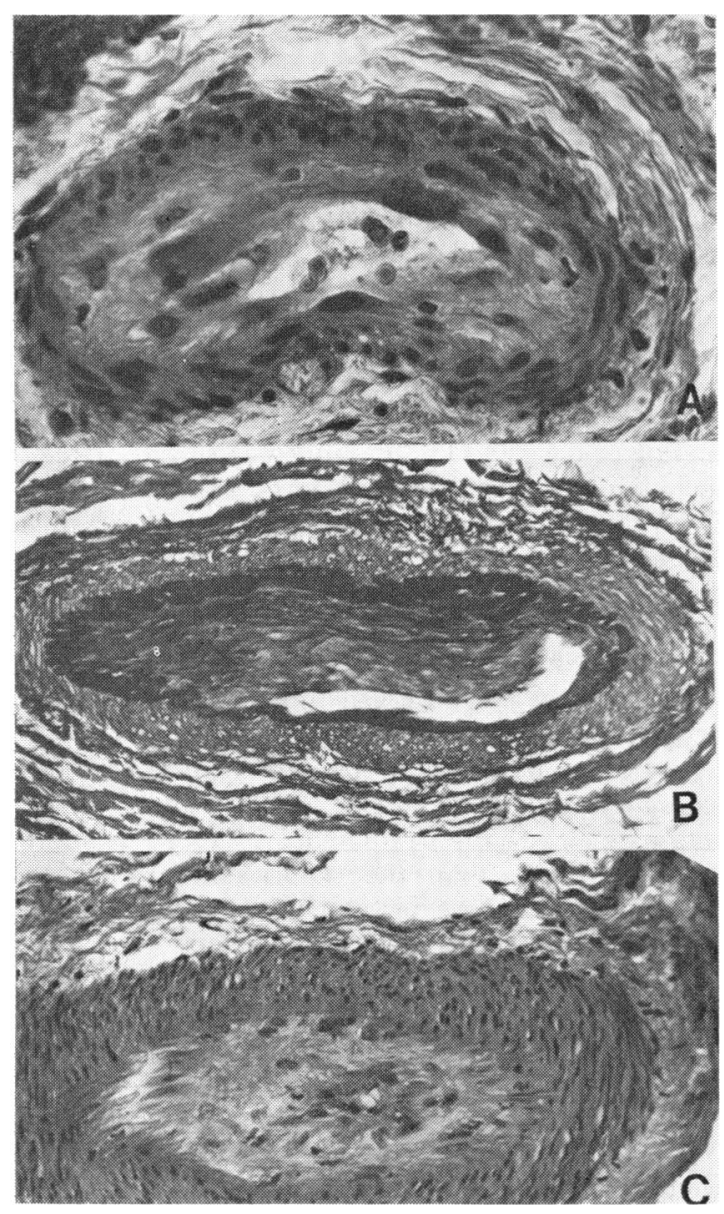

Fig. 4 Lesions in posterior ciliary arteries 6 months to 4 years after exposures of at least 6000 rads.

A: Concentric proliferation of intima in a small artery. Notice large and prominent endothelial cells.

(Haematoxylin and eosin, $\times 300$ ). B: Eccentric myointimal proliferation in large artery with thickening and duplication of elastic membrane. Notice marked reduction in lumen. (Verhoeff elastic-Van Gieson, $\times 124)$. C: Severe concentric myointimal proliferation which has reduced the lumen of this large artery to a minute central channel. (Haematoxylin and eosin, $\times 124)$

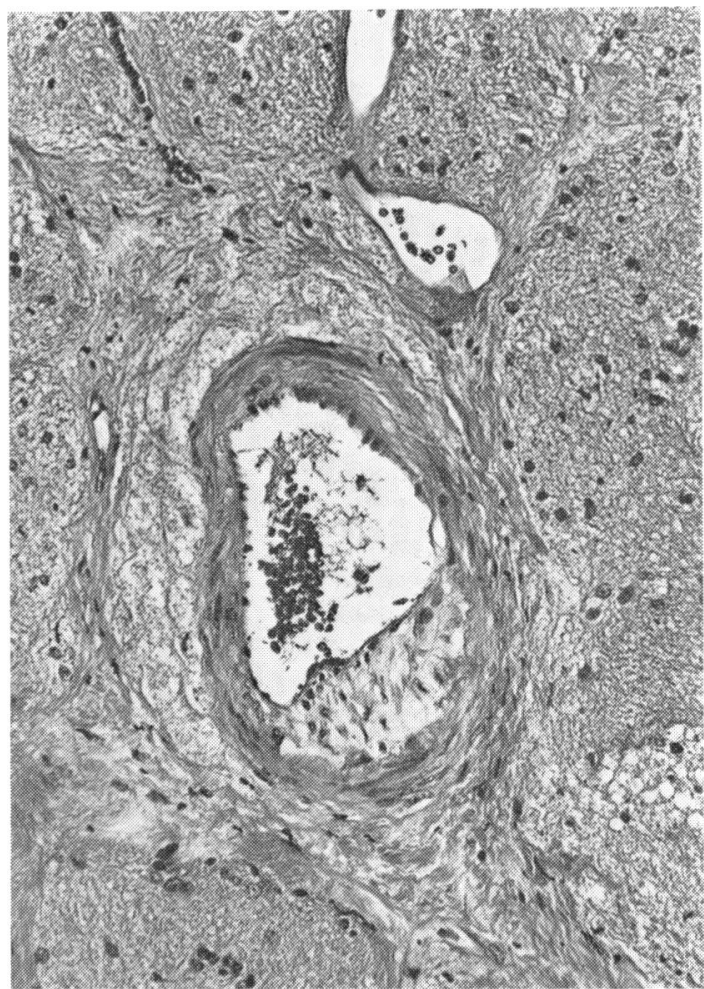

Fig. 5 Perpendicular section of optic nerve showing central retinal artery. There is an intimal plaque in the right lower quadrant. The vein (above) and the surrounding optic nerve are unremarkable in this field. (Haematoxylin and eosin, $\times 160$ ).

found that the ciliary arteries had developed significant alterations only a few months after standard therapeutic doses of radiation. Varying degrees of myointimal proliferation were found in most of the eyes that had been irradiated and none of the eyes that had not been irradiated. Conventionally it has been thought that the most radiosensitive ocular vessels are those of the retina. ${ }^{5}$ Our findings indicate the importance of damage to nonretinal as well as retinal vessels. Although histology alone is not a reliable indicator of blood flow, the severity of some of these changes raises the question of possible functional embarrassment of the posterior ciliary circulation, with ischaemia of the choroid and anterior optic nerve.

Previous histology of radiation-induced retinal vascular changes has shown thickening, hyalinisation, and occlusion. ${ }^{6}$ Retinal vascular changes observed with the aid of fluorescein angiography include capillary closure, vessel leakage, microaneurysms, and telangiectasis. ${ }^{57}$ The histological findings of occluded vessels explain capillary closure, 


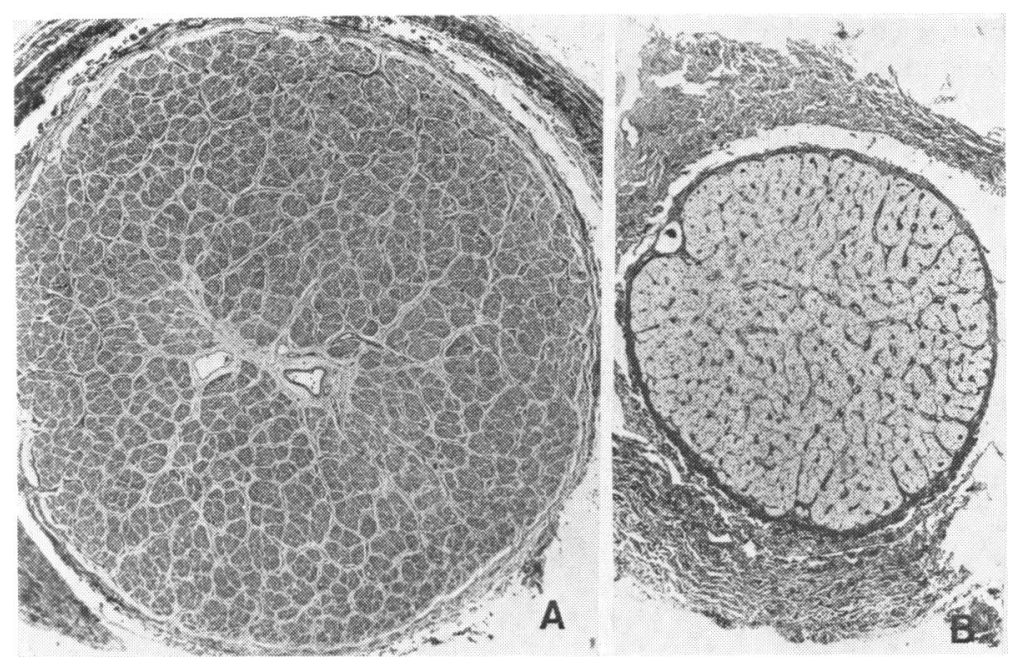

Fig. 6 Diffuse atrophy of optic nerve. A: Perpendicular section of normal optic nerve (child). The myelinated fascicles appear dark grey (bright blue in the preparation) and uniform. Compare with the adjacent photograph obtained at the same magnification and using the same stain.

B: Perpendicular section of optic nerve from eye resected 17 months after 6000 rads $/ 20$ fractions $/ 35$ days. Notice severe reduction in size and total, diffuse loss of myelin. (Luxol fast blue-PAS, $\times 17)$.

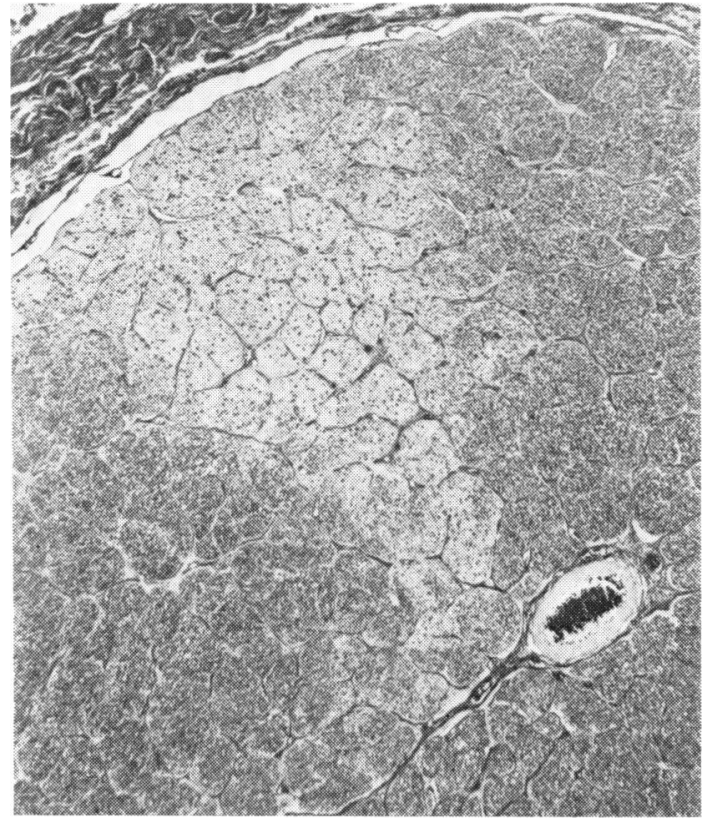

Fig. 7 Segmental atrophy of optic nerve from a nonirradiated eye containing retinoblastoma. In this perpendicular section the nerve fibres (shown as fine dark dots at right, lower and left margins) are absent in a triangular segment (pale area in centre and upper left). (Bielschowsky silver impregnction, $\times 42)$. and the extravascular fibrin we observed is an indication of vascular leakage. Fibrin deposition should not be unexpected because it is a typical finding of delayed radiation injury in several tissues, including brain, ${ }^{8}$ pericardium, ${ }^{9}$ and subcutis. $^{10}$ Central retinal artery occlusion is one of the possible complications of high-dose irradiation which has been clinically observed 1 to 2 years after treatment. ${ }^{3}$ The lesion of the central retinal artery is a myointimal proliferation which resembles the ciliary vessel changes we observed more than the retinal vessel changes.

Optic atrophy was a frequent feature in both irradiated and nonirradiated eyes. Although there was a tendency for irradiated eyes to show more diffuse atrophy than the nonirradiated eyes, an examination of a greater number of eyes would be necessary to prove this relationship. Radiation necrosis of the central nervous system is a wellknown phenomenon and occurs more readily in white matter than grey matter. ${ }^{8}$ The damage is most probably mediated through vascular changes.11 Optic atrophy has been observed after high doses of radiation, over 6800 rads $^{312}$ or after lower doses when combined with chemotherapy. ${ }^{1314}$

Our study has not demonstrated a clear time, dose, or age dependency of the ciliary artery lesions. Such an effect might be demonstrable either in a larger series of cases or in a prospective study having a wider range of radiation doses. Probably most of our patients have received doses above the threshold of arterial injury.

Radiation injury may take various forms and will vary from organ to organ, though it is usually consistent in a given organ. In the eye as in other tissues, with the notable exception of the liver and 


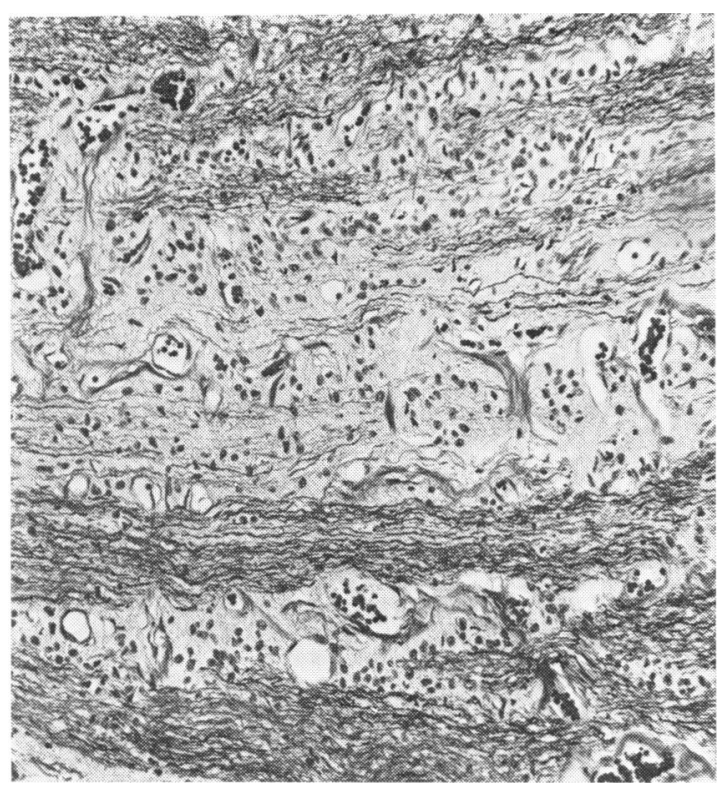

Fig. 8 Segmental atrophy of optic nerve from tumour-containing, nonirradiated eyes. Normal axons are shown as fascicles of wavy black lines at the bottom. The axons are absent in the centre and scanty at the top. (Bielschowsky silver impregnation, $\times 115)$.

intestine, there is a striking lack of lesions in ocular veins and sinusoids. ${ }^{10}$ Two features of the ocular changes are different from those found in other organs and deserve a comment: the irregular thickening of the retinal arterioles by a fine fibrillary material appears to be characteristic, since it is uncommon in most other tissues; unlike in many other organs and tissues in which fibrosis (i.e., collagen deposition) is typical of delayed radiation injury, we did not detect fibrosis of the choroid or sclera, even in the presence of severe narrowing of ciliary vessels.

We were surprised at the frequency of the vascular changes in the ciliary arteries and central retinal artery, occurring relatively soon after irradiation. If progressive, these changes could cause visual loss, either from central retinal artery occlusion, anterior ischaemic optic neuropathy, or choroidal ischaemia. Yet in a group of nonenucleated eyes in patients managed by the same method of radiotherapy and followed up for a mean of 11 years for visual function ischaemic changes were rare. Only 2 of 22 eyes had clinical optic nerve ischaemia, 1 macular ischaemia, and none central retinal artery occlusion. ${ }^{4}$ We do not know the reason for the discrepancy between the histopathological and clinical findings. One possible explanation is that radiation injury progresses rapidly for a limited time and then stabilises or progresses slowly.

Mrs Susan Lauber, Mrs Mia Hinze, and Mrs Irma Stadlbauer prepared the histological sections. Mr Jeff Shyshka prepared the photomicrographs.

This study was supported in part by grant 2 HUD 626 from the American Cancer Society and by Veterans Administration Research Funds MRIS 2735-01.

\section{References}

${ }^{1}$ Merriam GR Jr, Focht EF. A clinical and experimental study of the effect of single and divided doses of radiation on cataract production. Trans Am Ophthalmol Soc 1962; 60: $36-51$.

${ }^{2}$ Duke-Elder S, MacFaul PA. System of Ophthalmology. Non-mechanical injuries. St Louis: Mosby, 1978: 14 (2): 976-85.

${ }^{3}$ Shukovsky LJ, Fletcher GH. Retinal and optic nerve complications in a high dose irradiation technique of ethmoid sinuses and nasal cavity. Radiology 1972; 104: 629-34.

${ }^{4}$ Egbert PR, Donaldson SS, Moazed K, Rosenthal AR. Visual results and ocular complications following radiotherapy for retinoblastoma. Arch Ophthalmol 1978; 96: 1826-30.

${ }^{5}$ Hayreh SS. Post-radiation retinopathy: A fluorescence fundus angiographic study. $\mathrm{Br} J$ Ophthalmol 1970; 54: $705-14$.

${ }^{6}$ Howard GM. Ocular effects of radiation and photocoagulation. Arch Ophthalmol 1966; 76: 7-10.

${ }^{7}$ Chee P H Y. Radiation retinopathy. Am J Ophthalmol 1968; 66: 860-5.

${ }^{8}$ Rubinstein LJ. Tumors of the central nervous system. Atlas of Tumor Pathology, Fascicle 6 (2nd series). Washington, DC: Armed Forces Institute of Pathology, 1972: 349-60.

${ }^{9}$ Fajardo LF, Stewart JR. Experimental radiation-induced heart disease. I. Light microscopic studies. Am J Pathol 1970; 59: 299-316.

${ }^{10}$ Fajardo LF, Berthrong M. Radiation injury in surgical pathology. Part I. Am J Surg Pathol 1978; 2: 159-99.

${ }^{11}$ Haymaker W, Ibrahim MZM, Miguel J, Call N, Riopelle AJ. Delayed effects in the brains of monkeys exposed to $x$ and gamma rays. $J$ Neuropathol Exp Neurol 1968; 27: 50 79.

${ }^{12}$ Ross HS, Rosenberg S, Friedman AH. Delayed radiation necrosis of the optic nerve. Am J Ophthalmol 1973; 76: 683-6.

${ }^{13}$ Fishman ML, Bean SC, Cogan DG. Optic atrophy following prophylactic chemotherapy and cranial radiation for acute lymphocytic leukemia. Am J Ophthalmol 1976; 82: 571-6.

${ }^{14}$ Chan RC, Shukovsky LJ. Effects of irradiation on the eye. Radiology 1976; 120: 673-5. 